\title{
Desenvolvimento da Acuidade Visual de Grades
}

\author{
Solange Rios Salomão ${ }^{1}$ \\ Universidade Federal de São Paulo - UNIFESP
}

\begin{abstract}
Uma das funções visuais mais estudadas durante os primeiros anos de vida é a acuidade visual. A acuidade visual reflete a capacidade do sistema visual em discriminar detalhes de um objeto. Em bebês e pacientes não-verbais, utiliza-se a avaliação da acuidade visual de resolução de grades. Neste artigo, são mostrados os diferentes métodos de medida da acuidade de grades disponíveis para uso em clínica, juntamente com dados de desenvolvimento normal dessa função.
\end{abstract}

Descritores: Visão. Acuidade visual. Potenciais visuais evocados.

Tossas interações com o mundo externo são primariamente visuais, e o sentido da visão teve um papel fundamental no longo curso da evolução humana. A visão é um sentido mais importante para o ser humano do que para a maioria das espécies. A razão é que ela fornece informação espacial precisa de distância, dando informações altamente confiáveis sobre a localização e as propriedades de objetos no ambiente (Hurvitch, 1981; Shimojo, Paradiso \& Fujita, 2001).

Durante a infância, a experiência visual informa sobre o ambiente e também esculpe a estrutura visual do cérebro. A medida de funções visuais em bebês e crianças pré-verbais é uma tarefa que requer habilidades e métodos específicos. Uma limitação óbvia é que, diferentemente dos adultos, os bebês não podem seguir instruções ou dar respostas verbais.

A acuidade visual (AV) é definida como a habilidade do sistema visual em distinguir detalhes finos de objetos apresentados no espaço, ou seja, a medida do menor ângulo formado entre os detalhes de um determinado objeto

1 Professor Associado Livre-docente, Chefe do Laboratório de Eletrofisiologia Visual, Clínica do Departamento de Oftalmologia da Universidade Federal de São Paulo, Escola Paulista de Medicina. Endereço eletrônico: ssalomao@ oftalmo.epm.br 


\section{Solange Rios Salomão}

e sua imagem na retina. Esse ângulo é chamado de mínimo ângulo de resolução (MAR). Por exemplo, na consulta oftalmológica, o médico oftalmologista apresenta letras (ou símbolos, ou números) de diferentes tamanhos impressas numa tabela ou projetadas numa tela, e solicita ao paciente que as leia, em escala decrescente de tamanho, até o ponto em que não possa mais distinguir os detalhes do objeto apresentado (Cruz \& Salomão, 1998).

A medida da acuidade visual em adultos ou em indivíduos que conseguem informar o quanto enxergam é simples e direta, independente do modelo de tabela que se empregue. Existem diferentes tipos de acuidade visual. O mais conhecido de todos é a acuidade de reconhecimento, a habilidade de identificar corretamente letras ou formas, que se mede tipicamente com uma tabela de letras, como a conhecida tabela de Snellen.

Pode-se solicitar a crianças pequenas que, em vez de ler as letras do alfabeto, combinem letras ou padrões a uma amostra - como, por exemplo, os cartões de Lea, os cartões de Allen, o teste HOTV e a tabela do "E". No entanto, bebês não podem ser testados prontamente para esse tipo de acuidade. Em pacientes não-verbais, como bebês, crianças e adultos com distúrbios de comunicação (pacientes com múltiplas deficiências e/ou com deficiência neurológica), a medida da acuidade visual deve ser feita de forma objetiva. Os métodos objetivos implicam técnicas de medida da acuidade que independem da resposta verbal dos pacientes.

Para obter medidas das respostas perceptuais e sensoriais de bebês e crianças pré-verbais, foi necessário o desenvolvimento de técnicas específicas. As primeiras tentativas de quantificação da acuidade visual basearam-se na habilidade do bebê em detectar listras num tambor de nistagmo optocinético. O examinador notava a presença ou ausência do nistagmo pela observação direta dos movimentos oculares do bebê (Gorman, Cogan, \& Gellis, 1957). A partir da década de 1970, pesquisadores introduziram duas técnicas para definir o desenvolvimento da visão em bebês e crianças pré-verbais (Dobson \& Teller, 1978). Esses dois métodos - olhar preferencial (OP ou OP de escolha forçada) e potenciais visuais evocados (PVE) - ajudaram a nossa compreensão do desenvolvimento normal e anormal (Day, 1997).

A habilidade do ser humano em interpretar cenas visuais e reconhecer objetos pode ser examinada considerando-se a resolução de variações de lu- 


\section{Desenvolvimento da Acuidade Visual de Grades}

minância no espaço, pela medida da acuidade visual de resolução de grades (Skoczenski \& Norcia, 2002). A acuidade de grades em adultos é limitada pelo espaçamento dos cones na fóvea e pela função de borramento da óptica do olho. Os limites de amostragem, tanto ópticos como dos fotorreceptores, contribuem para o nível de detalhes espaciais que podem ser transmitidos para o resto do sistema visual.

Tanto o método comportamental (OP) como o método eletrofisiológico (PVE) de medida da acuidade visual se baseiam na habilidade do sujeito em resolver padrões listrados ou em formato de tabuleiro de xadrez. Essa tarefa visual é chamada de acuidade de resolução de grades. Vale ressaltar que na medida da acuidade por tabelas de optotipos, o tipo de tarefa que se exige é o de acuidade de reconhecimento, em que o sujeito deve reconhecer um símbolo e emitir uma resposta verbal para informar o quanto enxerga. As grades usadas como estímulos de acuidade podem ser listras pretas e brancas com bordas marcadas (grades de onda quadrada) ou grades senoidais - aquelas com perfil de luminância de onda senóide, que parecem listras com bordas borradas ou graduadas. Em ambos os casos, o "ciclo de trabalho" das grades é 1:1, ou seja, as barras escuras e as claras têm a mesma largura.

A unidade de AV mais comum é a notação de Snellen, que define a acuidade adulta normal como 20/20. Os valores de Snellen são comumente usados para determinar a acuidade de reconhecimento, mas outras duas unidades - mínimo ângulo de resolução e ciclos por grau de ângulo visual - são mais adequadas e utilizadas para medir a acuidade de grades. Todas essas unidades são baseadas ao definir o ângulo visual subentendido pelo menor estímulo que o sistema visual pode "resolver". Sem considerar as unidades usadas, os valores de acuidade dependem criticamente da distância do estímulo de acuidade em relação ao observador. À medida que aumenta a distância de um dado estímulo, seu ângulo visual diminui; ao contrário, um estímulo pequeno próximo do observador subentenderá o mesmo ângulo visual que um estímulo duas vezes maior no dobro da distância. Para grades de onda quadrada, o ângulo mínimo de resolução é definido como a menor separação detectável entre as linhas na grade que, dado um ciclo de 1:1, iguala-se à menor largura de listra detectável. Abaixo do limiar de resolução, as listras não podem ser individualmente percebidas e se juntam num cinza homogêneo. 


\section{Solange Rios Salomão}

O mínimo ângulo de resolução é expresso em minutos de arco de ângulo visual, com 1 minuto de arco representando a acuidade adulta normal. Grades senoidais e quadradas podem ser definidas em termos de sua freqüência espacial, medida pelo número de ciclos da grade dentro de um grau de ângulo visual. Um ciclo equivale a uma barra escura mais uma barra clara da grade, ou duas vezes a largura de uma única barra usada para definir o mínimo ângulo de resolução. Sendo assim, um ângulo de resolução de 1 minuto de arco corresponde a uma frequiência espacial de 30 ciclos por grau de ângulo visual $(1$ ciclo $=2$ minutos de arco $=30$ ciclos $/ g r a u)$. Portanto, a acuidade crescentemente melhor é representada por valores progressivamente menores do mínimo ângulo de resolução, mas por freqüências espaciais maiores.

As medidas de acuidade visual obtidas por essas técnicas podem, muitas vezes, subestimar a verdadeira capacidade do sujeito de executar a tarefa visual que lhe está sendo exigida. Isto se deve à presença inevitável de "ruído" em todos os testes, sejam eles comportamentais ou eletrofisiológicos. Existem muitas fontes de "ruído" que podem interferir nas medidas de desempenho visual: ruído comportamental - cooperação, mudanças no estado geral, fatores de atenção; ruído óculo-fisiológico - acomodação imprecisa, imaturidade no controle da movimentação dos olhos; ruído externo - interferência elétrica em registros eletrofisiológicos em função da atividade muscular, ruído inerente ao estímulo luminoso em si e ruído neurofisiológico - ruído inerente à transmissão dos sinais visuais ao longo da via visual a partir dos fotorreceptores até o local em que a resposta visual está sendo medida (Hamer \& Mayer, 1994).

\section{Olhar preferencial}

A técnica que proporcionou o maior número de dados comportamentais nas últimas três décadas é a do "olhar preferencial de escolha forçada" (OPEF ou FPL), além de suas variantes "olhar preferencial operante" (OPO ou OPL) e procedimento dos cartões de acuidade de Teller (CAT ou TAC).

A resposta de olhar preferencial foi descrita por Robert Fantz (1958), um psicólogo estudioso do desenvolvimento humano, para examinar a discriminação de bebês a diversos estímulos visuais (Fantz, 1958; Fantz, Ordy, \& Udelf, 1962). A resposta de olhar preferencial, que foi descrita por Fantz, diz respeito 


\section{Desenvolvimento da Acuidade Visual de Grades}

à preferência natural do recém-nascido a olhar para um estímulo estruturado (listras pretas e brancas) quando comparado a um estímulo homogêneo. As técnicas de olhar preferencial (OP ou PL) se baseiam na tendência que os bebês demonstram em fixar uma superfície com padrões em preferência a uma superfície homogênea de igual luminância (Birch, 1989; Mackie, Saunders, Day, Dutton, \& Mcculloch, 1996). As preferências dos bebês a estímulos específicos foram inferidas a partir de diferenças no comportamento visual, tais como tempo de fixação mais longo em um dos estímulos quando um par era mostrado.

O olhar preferencial de escolha forçada (OPEF) foi desenvolvido por Teller (Teller, 1979; Teller, Morse, Borton, \& Regal, 1974), combinando o paradigma do olhar preferencial descrito por Fantz com princípios de métodos psicofísicos objetivos, usando-se estímulos quantificáveis. Os estímulos utilizados são estímulos de grades (listras brancas e pretas) de onda quadrada de alto contraste, pareados com um estímulo cinza homogêneo de mesma luminância espacial média. $\mathrm{O}$ estímulo de grade está posicionado eqüidistante à direita ou à esquerda de um orifício de observação central pelo qual um adulto observa o bebê. Um observador adulto que desconhece a localização e a frequiência espacial da grade faz um julgamento de escolha-forçada sobre a localização esquerda ou direita da grade em cada apresentação. Os julgamentos do observador são baseados na totalidade das respostas visuais do bebê, em vez de confiar somente num aspecto do comportamento visual infantil, por exemplo, o tempo de fixação.

A função psicométrica é derivada da porcentagem de respostas corretas que o observador fez, baseado em várias grades sucessivas que são apresentadas para cobrir a faixa compreendida entre o acaso (50\% num OPEF de 2 alternativas) e 100\% de respostas corretas. A acuidade do bebê é definida como a freqüência espacial da grade que corresponde ao desempenho de porcentagem de respostas corretas do observador é estatisticamente significante acima do acaso (tipicamente, $75 \%$ ). O procedimento de OPEF emprega uma metodologia psicofísica rigorosa, e a situação experimental é montada de forma a impedir que o experimentador interaja com a criança. A criança é sentada diante de um painel cinza no qual se apresentam dois estímulos: uma grade e um cinza de igual luminância média, cujas localização e frequiência espacial são aleatoriamente determinadas segundo o método dos estímulos constantes 


\section{Solange Rios Salomão}

ou outros métodos psicofísicos (Lewis \& Maurer, 1986; Mayer, Fulton, \& Hansen, 1982). A introdução de métodos psicofísicos representou um avanço indiscutível na confiabilidade e precisão da medida da acuidade visual, mas implicou um tempo de testagem longo (aproximadamente 1 hora), o que inviabilizou o OPEF como método de testagem clínica. Algumas modificações no número de apresentações dos estímulos e o emprego de ambientes isentos de distrações, como salas totalmente escurecidas e estímulos projetados, puderam reduzir o tempo de teste para aproximadamente 10 a 15 minutos (Birch \& Hale, 1988). No entanto, o uso de estímulos projetados encarece e restringe o equipamento, que deve ser montado em ambiente totalmente escurecido e que não é portátil. Portanto, seu uso em clínica é restrito.

A técnica do olhar preferencial operante (OPO) foi desenvolvida porque os testes de OPEF eram muito entediantes para crianças a partir dos 15 meses de idade (Birch, 1989). Nessa faixa etária, a transformação do teste em um tipo de jogo de "apontar" ajuda a manter o interesse da criança. Para cada resposta correta que o sujeito emitir (apontando para a grade ao invés de apontar para o campo cinza homogêneo), um reforço lhe é dado. $\mathrm{O}$ reforço pode ser algo para comer (sucrilhos ou pequenas balas), um aplauso ou um cumprimento verbal. A promessa de um brinquedo ou qualquer outro presente ao fim do teste também pode ser um auxílio com crianças a partir dos 24 meses de idade. O termo OPO tem sido usado para descrever OPEF associado a reforços para respostas corretas (Birch \& Hale, 1988; Mayer \& Dobson, 1982).

\section{Teste dos Cartões de Acuidade de Teller}

Visando fornecer testes mais adequados para uso clínico, o teste do OPEF, que tinha um protocolo estatístico rigoroso para determinar o limiar de acuidade visual, foi modificado, e criou-se o teste dos cartões de acuidade (McDonald et al., 1985). Esse teste recebeu o nome de Teste dos Cartões de Acuidade de Teller em homenagem à pesquisadora americana Davida Teller, em reconhecimento de sua dedicação a estudos do desenvolvimento da acuidade visual em bebês com a utilização do paradigma do olhar preferencial e, também, pelo fato de o teste dos cartões de acuidade ter sido desenvolvido em seu laboratório. 


\section{Desenvolvimento da Acuidade Visual de Grades}

O teste dos Cartões de Acuidade de Teller (CAT) utiliza como equipamento um jogo de cartões de papelão com as dimensões de $25,5 \times 47,5 \mathrm{~cm}$ na cor cinza. Cada cartão possui, em um dos lados, uma grade de onda quadrada de $12,5 \times 12,5 \mathrm{~cm}$ (com orientação vertical) reproduzida fotograficamente e colada num fundo de luminância média equivalente. O jogo completo é composto de 17 cartões com frequiência espacial variando de 0,23 a 38 ciclos/grau em intervalos de 0,5 oitava e contraste de $82 \%$, além de um cartão cinza sem listras para utilização como controle. No centro de cada cartão, há um orifício circular pelo qual o examinador observa as reações do paciente. Com a utilização de três distâncias (38, 55 e $84 \mathrm{~cm}$ ), é possível testar acuidades desde 20/2000 até 20/11. A iluminação exigida para a execução do teste é de 10 candelas $/ \mathrm{m}^{2}$. Para evitar distrações durante o exame, pode-se usar um painel de madeira cinza que funciona como um anteparo para a apresentação dos cartões.

A diferença mais importante entre o CAT e os procedimentos tradicionais do OPEF é a natureza dos julgamentos feitos pelo examinador. No CAT, o julgamento da localização da grade é se baseia na qualidade e na coerência do comportamento de olhar da criança, para certificar que ela consegue discriminar aquela grade. Quando as listras na grade são largas e o comportamento de olhar da criança se dirige claramente àquela grade, o julgamento é normalmente baseado em duas apresentações daquela grade, com a posição direita-esquerda da grade revertida entre as duas apresentações. À medida que o limite da acuidade se aproxima, o comportamento de olhar da criança tornase menos coerente, e o examinador pode precisar mostrar a grade várias vezes para determinar se a criança está mostrando olhar preferencial em direção à grade. No OPEF, a tarefa do examinador é usar o comportamento de olhar da criança para julgar se a grade está à direita ou à esquerda. $\mathrm{O}$ examinador deve, obrigatoriamente, emitir um julgamento (esquerda ou direita) em cada apresentação do estímulo, mesmo que esteja em dúvida. Daí a denominação escolha forçada. O examinador do CAT, por outro lado, usa a resposta global da criança (apontar, dirigir a cabeça, virar o corpo, verbalizar, além de dirigir o olhar) e pode interagir com a criança para fazer um julgamento subjetivo sobre a grade mais fina que ela consegue ver. Isto permite maior agilidade e flexibilidade, tornando o método aplicável para a situação clínica. 


\section{Solange Rios Salomão}

\section{Desenvolvimento da acuidade visual de resolução de grades pelo teste dos}

cartões de teller

Definido o valor de acuidade visual para um determinado teste, é fundamental comparar os resultados obtidos com normas descritas na literatura. Dessa forma, a acuidade pode ser classificada como normal para a idade do paciente, de acordo com valores da média da população e com o mínimo valor esperado para considerar-se normal (limite normal inferior). Valores normativos e de desenvolvimento normal para testagem da acuidade monocular e binocular estão disponíveis a partir de dois estudos populacionais (Salomão \& Ventura, 1995; Mayer et al., 1995), sendo que as normas descritas em um dos estudos citados foram totalmente elaboradas no Brasil (Salomão \& Ventura, 1995). O estudo brasileiro foi feito com 641 crianças de baixa renda do município de São Paulo. As normas são mostradas na Figura 1.

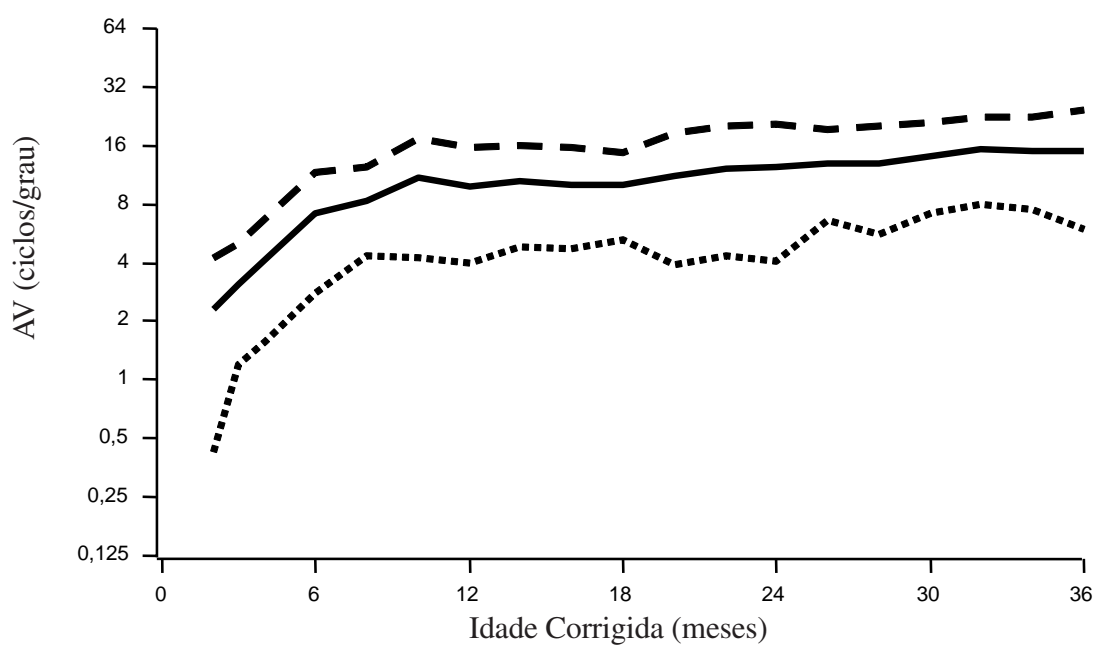

Figura 1. Média da acuidade visual de grades monocular (linha sólida) e limites de tolerância inferior (linha pontilhada pequena) e superior (linha pontilhada grande) para $90 \%$ da população, com probabilidade de $95 \%$, com base em dados de 624 bebês e crianças saudáveis em função da idade. Reproduzido de Salomão e Ventura, 1995, sob permissão. 


\section{Desenvolvimento da Acuidade Visual de Grades}

\section{Potenciais visuais evocados}

O potencial visual evocado (PVE) é uma pequena resposta elétrica do cérebro que ocorre de forma estereotipada após a apresentação de um estímulo visual como, por exemplo, a reversão de contraste em um padrão do tipo tabuleiro de xadrez ou de grades ou um flash de luz (Brigell, 2001). O registro do PVE é relativamente simples e totalmente não invasivo, em função da proximidade do córtex visual à superfície do couro cabeludo (Harding, 1991). Estudos neurofisiológicos em córtices de macacos mostram que os PVEs têm origem nas camadas mais superficiais do córtex visual (Schroeder, 1991). Esses potenciais são da ordem de microvolts e são obscurecidos pelas flutuações de voltagem muito maiores do eletroencefalograma (EEG) (Norcia, 1994).

O registro do PVE é feito com eletrodos de cúpula de prata ou ouro preenchidos com gel condutor e colocados sobre o couro cabeludo após limpeza prévia da pele. Os eletrodos devem ser posicionados de acordo com o

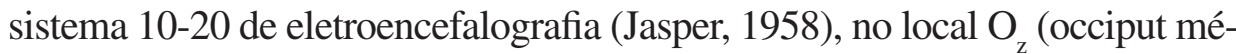
dio), acrescido ou não - dependendo da investigação topográfica na via visual - de eletrodos $\mathrm{em}_{3}$ (occiput esquerdo) e $\mathrm{O}_{4}$ (occiput direito). A amplitude do $\operatorname{PVE}(3-25 \mu \mathrm{V})$ é muito menor do que a do EEG, que gira em torno de $100 \mu \mathrm{V}$. Em virtude dessa diferença, faz-se necessário o registro de várias estimulações (no mínimo 80), e a média dos registros é calculada.

O PVE reflete primariamente a atividade elétrica gerada pela estimulação do campo visual central. Existem três razões para o predomínio do campo visual central na resposta do PVE: 1) o mapa retinotópico do córtex estriado em que a representação do campo visual central está localizada mais próxima à superfície no pólo occipital, visto que a representação do campo periférico está mais profundamente localizada dentro do sulco calcarino; 2) a projeção foveal é magnificada no córtex (Horton \& Hoyt, 1991); 3) estímulos de tabuleiro de xadrez pequenos podem ser resolvidos somente pelo campo visual central (Brigell, 2001).

Os estímulos mais preconizados para o registro do PVE são padrões em forma de tabuleiro de xadrez ou grades com listras brancas e pretas de alto contraste com reversão de padrão (alternante), de forma que as áreas iluminadas (brancas) se tornam escuras (pretas) e as áreas escuras se tornam ilumina- 


\section{Solange Rios Salomão}

das. Esse tipo de estimulação gera PVEs confiáveis na maioria dos pacientes. O PVE pode ser registrado sob condições de estímulos transientes ou de estado estável. O PVE transiente é produzido quando há um intervalo inter-estímulos suficiente ( $<2 \mathrm{~Hz}$ ou 4 alternâncias/s) para que o cérebro resgate seu estado de repouso, ou seja, com freqüência temporal mais lenta. O PVE de estado estável é produzido quando o cérebro não resgata seu estado de repouso, ou seja, os estímulos têm uma taxa de repetição mais rápida, geralmente de 4 a $10 \mathrm{~Hz}$ ou 8 a 20 alternâncias/s (Skarf, 1989).

O PVE registrado da superfície do couro cabeludo e sincronizado com as transições no estímulo visual indica a presença de uma resposta neural a um estímulo que prescinde da resposta verbal ou comportamental de detecção. Por esse motivo, o PVE tem sido um método atraente na pesquisa da visão infantil ou em pacientes em que as respostas motoras e a comunicação verbal são ausentes ou limitadas (Atkinson, 2000). A acuidade visual pode ser medida pelo PVE e é definida como o tamanho do elemento de um estímulo de padrões em que o PVE não pode mais ser provocado no córtex visual.

Respostas eletrofisiológicas da parte visual do cérebro ou potenciais visuais evocados (PVEs) são um método alternativo da avaliação da acuidade de resolução de grades (Fulton \& Robb, 1987). Os PVEs são sinais elétricos em massa gerados pelas áreas occipitais 17, 18 e 19 em resposta à estimulação visual. Quando os PVEs são registrados com estímulos de flashes de luz, a informação obtida diz respeito somente à integridade das vias visuais, uma vez que as respostas a esses estímulos não sofrem interferência de borramento visual. Portanto, a medida da acuidade visual pelos PVEs deve ser feita com estímulos de padrões listrados (grades) ou quadriculados (tabuleiros de xadrez) (Birch, 1989).

Os registros dos PVEs requerem equipamento considerável, incluindo amplificadores fisiológicos e um sistema de computador para gerar os estímulos e analisar as respostas. Contudo, a variabilidade das estimativas de acuidade pelo PVE é geralmente melhor do que as obtidas com os métodos comportamentais, de maneira que o método do PVE pode ser mais sensível para detectar pequenas diferenças de acuidade. Ambos os métodos dependem do estado de alerta e atenção do sujeito, e ambos requerem que o testador julgue precisamente se o sujeito está fixando e focalizando o estímulo. 


\section{Desenvolvimento da Acuidade Visual de Grades}

As respostas corticais a mudanças temporais nos padrões são somadas e podem fornecer uma estimativa da acuidade. Os PVEs medem a responsividade do córtex visual, mas são dependentes da via retino-cortical inteira. Assim, se um déficit de acuidade é produzido por disfunção retiniana, se refletirá na informação transmitida da retina ao córtex visual. A criança observa padrões num monitor de vídeo. Os sinais são levados dos eletrodos a um amplificador, e deste a um dispositivo capaz de calcular a média das respostas visualmente evocadas. Amplificadores, microprocessadores dedicados a calcular a média e sistemas que exibem padrões estão comercialmente disponíveis como sistemas integrados ou vendidos separadamente. Também se podem usar microcomputadores equipados com hardware apropriado para calcular a média dos sinais. A principal razão para calcular a média dos sinais e filtrá-los é melhorar a relação sinal-ruído (SNR). O PVE é contaminado por atividade do EEG, assim como por ruídos externos. No entanto, o PVE para um dado padrão de estimulação é razoavelmente constante em amplitude e latência (ou fase) enquanto a atividade do EEG ocorre ao acaso com respeito ao estímulo visual. Dessa forma, calculando a média da atividade do PVE para somá-la linearmente, a atividade do EEG diminui em proporção à raiz quadrada do número de PVEs mediados. Filtros de passagem alta e passagem baixa podem melhorar ainda mais a relação sinal-ruído (Cruz \& Salomão, 1998).

A amplitude revela o tamanho da onda registrada do pico da onda negativa $\left(\mathrm{N}_{1}\right)$ ao pico da onda positiva $\left(\mathrm{P}_{1}\right)$. A Figura 2 mostra o registro de um PVE por reversão de padrões de um sujeito adulto normal, com seus respectivos componentes. A latência reflete o tempo necessário para se alcançar o pico máximo da onda positiva. Esses parâmetros podem comparar-se a dados normativos de acordo com a idade do paciente (Marg, Freeman, \& Petzman, 1976; Sokol, 1978; Sokol, Moskowitz, Mccormack \& Augliere, 1988).

Na prática clínica, esse teste pode ser influenciado e contaminado por artefatos. Por exemplo, se a criança não fixar o estímulo por cooperação limitada, ou por movimentos anormais e nistagmóides dos olhos, os resultados obtidos podem ser espúrios (Friendly, Weiss, \& Barnet, 1986; Sokol, Hansen, Moskowitz, Grenfield, \& Towle, 1983). Outro ponto importante a salientar é que a maioria dos equipamentos comerciais de PVEs não são planejados para testagem de crianças - às vezes, torna-se necessário proceder a várias modi- 


\section{Solange Rios Salomão}

ficações. Para o registro dos PVEs em bebês, é importante manter a criança alerta e atenta ao estímulo. Utilizam-se para tanto pequenos brinquedos, que podem ser mostrados na frente do monitor de vídeo onde os estímulos são gerados durante o teste. Como os PVEs refletem a atividade macular, esses brinquedos devem ser muito pequenos ou abertos no centro de forma a não bloquear a visão do estímulo pela mácula. Também há o problema de que os bebês não podem ser instruídos a olhar para o padrão; assim, um observador deve olhar para a criança e sinalizar quando ela está fixando para que o registro seja feito nesse momento. Pedais e botões de mouse podem ser usados como interruptores para esse propósito. O equipamento que faz a média e a análise das ondas registradas deve conter software e hardware específicos para rejeitar possíveis artefatos e eliminar sua contribuição no registro médio final.

\section{Potenciais visuais evocados de varredura (PVEVs)}

A idéia do registro contínuo do PVE de estado estável durante a varredura rápida de uma faixa grande de estímulos foi proposta por Regan em 1973 (Regan, 1973). O método do PVE de varredura (PVEV) é um tipo de PVE por reversão de padrões de estado estável. A técnica foi aplicada pela primeira vez para medir a acuidade de resolução de grades (Tyler, Apkarian \& Levi, 1979). Um padrão listrado que se reverte em contraste é varrido de listras largas (freqüências espaciais baixas) a listras finas (frequiências espaciais altas) durante um período de 10 segundos. O PVEV foi desenvolvido para situações em que se obtêm somente curtos intervalos de atenção por parte do paciente, como acontece, por exemplo, com bebês ou crianças não-verbais. É relativamente fácil manter a atenção do bebê num nível constante durante um período de 10 segundos, ao contrário do que ocorreria num período de vários minutos (Norcia, 1994). Cada largura de grade (freqüência espacial) se apresenta por 1 segundo.

O sinal obtido é transformado por análise no computador para isolar a parte do sinal do PVE que ocorre naquela taxa de reversão por meio de modelo matemático da transformada de Fourier (Bach, 1999). Pela medida dos componentes do EEG em frequiências harmônicas às frequiências do estímulo, pode-se medir o PVE. Mudanças na amplitude e na fase dessa frequiência após 


\section{Desenvolvimento da Acuidade Visual de Grades}

a resposta podem ser medidas à proporção que se muda um parâmetro do estímulo, como o tamanho da listra ou o contraste. Para cada intervalo de 0,5 $\mathrm{s}$, a amplitude e a fase do sinal transformado são computadas. A acuidade é estimada por regressão linear e extrapolação à largura de listra que corresponde à amplitude de zero microvolt (Sokol, 1978). A técnica tem sido utilizada para a medida da acuidade de grades, e os resultados foram bem correlacionados com acuidades obtidas por métodos psicofísicos (Riddell et al., 1997; Tyler et al., 1979).

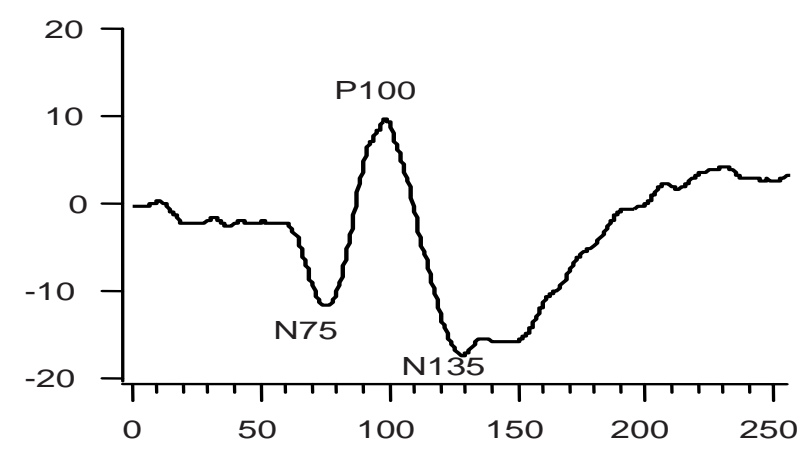

Figura 2. Registro do PVE por reversão de padrões de 15 ' de ângulo visual, obtido de voluntário saudável normal no Laboratório de Eletrofisiologia Visual Clínica da UNIFESP-Escola Paulista de Medicina. Notam-se os componentes N75, P100 e N135.

Os PVEVs combinam as vantagens de rápida apresentação dos estímulos - de forma que uma medida de acuidade de grades pode ser obtida numa única varredura de 10 segundos - com um eficiente sistema digital de análise dos sinais, que resulta em aumento da relação sinal-ruído (Norcia, Clarke, \& Tyler, 1985; Norcia \& Tyler, 1985). Por exemplo, para medir a acuidade de grades, a frequiência espacial de uma grade de onda-quadrada de alto contraste é varrida linearmente sobre uma taxa de 20:1, começando com uma grade 


\section{Solange Rios Salomão}

bem destacada ( 0,5 ciclos/grau) e terminando além do limite de resolução (40 ciclos/grau). Durante a apresentação de 10 segundos, a grade é rapidamente revertida em fase para gerar um PVE de estado estável. A varredura de freqüência espacial é ajustada de acordo com a idade do paciente.

Métodos modernos de análise de Fourier são usados para medir a amplitude dos PVEVs no segundo harmônico (por exemplo, $12 \mathrm{~Hz}$ para uma grade com alternância de fase de $6 \mathrm{~Hz}$ ). $\mathrm{O}$ valor da acuidade (em ciclos/grau) é estimado por extrapolação quando a amplitude da resposta alcança 0 microvolt (Figura 3). Normas de acuidade de resolução medida pelos PVEVs foram descritas (Norcia \& Tyler, 1985).

\section{Considerações sobre o desenvolvimento da $\mathrm{AV}$ de resolução}

A acuidade de grades se desenvolve nos primeiros anos de vida, e seu curso de desenvolvimento depende da técnica de medida usada. Dessa maneira, os valores de acuidade obtidos podem ser especificamente chamados de acuidade de OP e acuidade de PVE. As acuidades pelos PVEs tendem a ser mais altas do que as do OP na maior parte da infância. $\mathrm{O}$ achado de que a acuidade PVE é mais alta do que a OP pode ter a influência de vários fatores experimentais que contribuem para essa diferença: dentre eles, uso de estímulos piscantes, média de sinal, critérios de score generosos e somação de sinais no campo visual como um todo. Além disso, os PVEs são provavelmente controlados em grande parte pelos processos corticais precoces, visto que os dados comportamentais se baseiam na criança como um todo, incluindo os estágios mais tardios do processamento visual, assim como os do processamento central e motor (Teller, 1997).

A medida objetiva da acuidade visual em pacientes não-verbais tem sido incorporada gradativamente à rotina de exame oftalmológico, preenchendo uma importante lacuna e fornecendo informação valiosa sobre a visão desses pacientes. Além disso, o seguimento funcional de importantes doenças oftalmopediátricas pode ser quantitativamente avaliado, e novos protocolos de tratamento podem ser desenvolvidos e ter sua eficácia testada. Esses métodos também podem dar sua contribuição à decisão cirúrgica em casos duvidosos, como os de cataratas parciais, ptose, opacidades corneanas moderadas etc. 


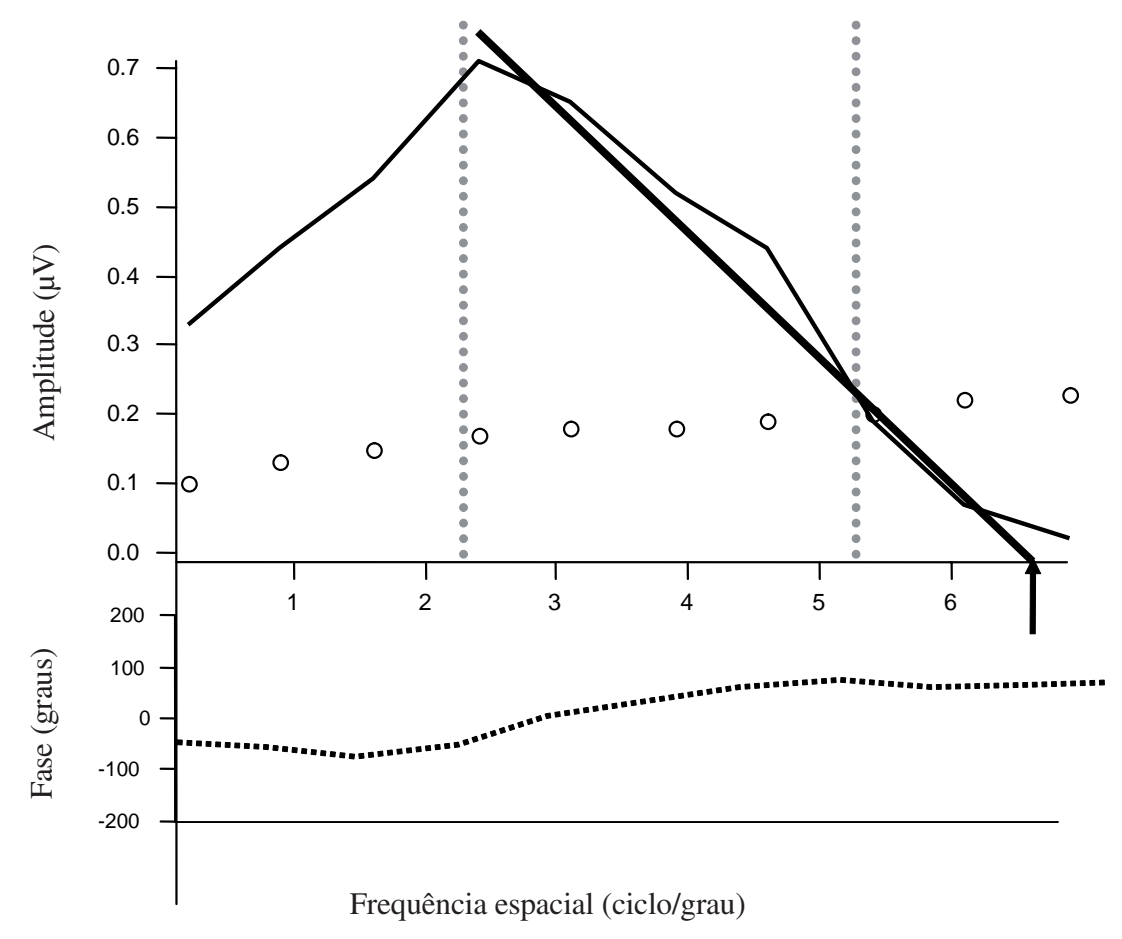

Figura 3. Análise de potencial visual evocado de varredura binocular de bebê saudável, nascido a termo, com 4 meses de idade, obtida no Laboratório de Eletrofisiologia Visual Clínica da UNIFESP-Escola Paulista de Medicina. A acuidade foi estimada como o intercepto em $0 \mu \mathrm{V}$ da reta de regressão linear que passa pelo pico da amplitude (linha sólida grossa) atingindo o eixo das freqüências espaciais. A seta verde no eixo das freqüências espaciais mostra o valor de 6,71 ciclos/grau ou 20/90, que corresponde ao limiar de acuidade visual de resolução de grades. 


\title{
Solange Rios Salomão
}

Salomão, S. R., (2007). Grating acuity development. Psicologia USP, $17(2), 63-81$.

\begin{abstract}
Visual acuity is one of the most studied visual functions during the first years of life. Visual acuity reflects the ability to resolve fine details from an observed object. Grating stimuli has been used in infants, pre-verbal and non-verbal patients to evaluate visual acuity. Clinical methods to measure grating acuity currently available along with normal developmental data of this function are presented.
\end{abstract}

Index terms: Vision. Visual acuity. Visual evoked potentials.

Salomão, S. R., (2007). Le developpement grinçant d'acuite. Psicologia USP, 17(2), 63-81.

Résumé: L'acuité visuelle est une des fonctions visuelles les plus étudiées chez les bébés ao cours des premières années de vie. Cette fonction reflète la capacité de résoudre de beaux détails d'un objet observé. Pour évaluer l'acuité visuelle, des stimuli ont été utilisés chez les bébés et chez les malades préverbaux et non-verbaux. On présent ici les méthodes cliniques actuellement disponibles pour mesurer l'acuité visuelle et aussi certaines données sur le développement normal de cette fonction.

Mots-clés: Vision. Acuité visuelle. Potentiels visuels évoqués.

\section{Referências}

Atkinson, J. (2000). Paediatric vision testing. In The developing visual brain (pp. 25-27). New York: Oxford University Press.

Bach, M. (1999). Do's and don'ts in Fourier analysis of steady-state potentials. Documenta Ophthalmologica, 99, 69-82.

Birch, E. E. (1989). Visual acuity testing in infants and young children. In D. G. Birch (Ed.), Ophthalmology clinics of North America (pp. 369-389). Philadelphia: WB Saunders. 


\section{Desenvolvimento da Acuidade Visual de Grades}

Birch, E. E., \& Hale, L. A. (1988). Criteria for monocular acuity deficit in infancy and early childhood. Investigative Ophthalmology and Visual Science, 29, 636-643.

Brigell, M. G. (2001). The visual evoked potential. In G. A. Fishman, D. G. Birch, G. E. Holder, \& M. G. Brigell (Ed.), Electrophysiologic testing in disorders of the retina, optic nerve and visual pathway (pp. 237-279). San Francisco: The Foundation of the American Academy of Ophthalmology.

Cruz, A. A. V., \& Salomão, S. R (1998). Acuidade visual. Arquivos Brasileiros de Psicologia, $50,9-26$.

Day, S. (1997). History, examination and further investigation. In D. Taylor (Ed.), Pediatric ophthalmology (pp. 79-82). Oxford: Blackwell Science.

Dobson, V., \& Teller, D. Y. (1978). Visual acuity in human infants: A review and comparison of behavioral and electrophysiological studies. Vision Research, 18, 1469-1483.

Fantz, R. L. (1958). Pattern vision in young infants. The Psychological Record, 8, 43-47.

Fantz, R., Ordy, J., \& Udelf, M. (1962). Maturation of pattern vision in infants during the first six months. Journal of Comparative and Physiological Psychology, 55, 907-912.

Friendly, D. S., Weiss, I. P., \& Barnet, A. B. (1986). Pattern-reversal visual evoked potentials in the diagnosis of amblyopia in children. American Journal of Ophthalmology, 102, 329-334.

Fulton, A. B., Robb, R. M. (1987). Special diagnostic and therapeutic modalities in pediatric ophthalmology. In Pediatric clinics of North America (pp. 1543-1553). Philadelphia: WB Saunders.

Gorman, J. J., Cogan, D. G., \& Gellis, S. S. (1957). An apparatus for grading the visual acuity of infants on the basis of optokinetic nystagmus. Pediatrics, 19, 1088-1092.

Hamer, R. D., Mayer, D. L. (1994). The development of spatial vision. In D. M. Albert \& F. A. Jakobiec (Eds.), Principles and practice of ophthalmology: Basic sciences (p p. 578608). Philadelphia: WB Saunders.

Harding, G. F. A. (1991). Visual evoked cortical potentials: Basic recording. In J. R. Heckenlively \& G. B. Arden (Eds.), Principles and practice of clinical electrophysiology of vision (pp. 399-407). St. Louis, MO: Mosby Year Book.

Horton, J. C., \& Hoyt, W. F. (1991). The representation of the visual field in human striate cortex: A revision of the classic Holmes map. Archives of Ophthalmology, 109, 816-824.

Hurvich, L. M. (1981). Color vision and its deficiencies. Impact of Science on Society, 31(2), 151-164.

Jasper, H. H. (1958). The ten twenty electrode system of the International Federation. Electroencephalography and Clinical Neurophysiology, 10, 371-380. 


\section{Solange Rios Salomão}

Lewis, T. L., \& Maurer, D. (1986). Preferential looking as a measure of visual resolution in infants and toddlers: A comparison of psychophysical methods. Child Development, 57, 1062-1075.

Mackie, R. T., Saunders, K. J., Day, R. E., Dutton, G. N., \& Mcculloch, D. L. (1996). Visual acuity assessmetn of children with neurological impairment using grating and vanishing optotype acuity cards. Acta Ophthalmologica Scandinavica, 74, 483-487.

Marg, E., Freeman, D. N., \& Petzman, P. (1976). Visual acuity development in human infants: Evoked potential measurements. Investigative Ophthalmology, 15, 150-157.

Mayer, D., \& Dobson, V. (1982). Visual acuity development in infants and young children as assessed by operant preferential looking. Vision Research, 11, 1141-1144.

Mayer, D. L., Fulton, A. B., \& Hansen, R. M. (1982). Preferential looking acuity obtained with a staircase procedure in pediatric patients. Investigative Ophthalmology and Visual Science, 23, 538-543.

Mayer, D. L., Beiser, A. S., Warner, A. F., Pratt, E. M., Raye, K. N., \& Lang, J. M. (1995). Monocular acuity norms for the Teller acuity cards between ages one month and four years. Investigative Ophthalmology and Visual Science, 36, 671-685.

Mcdonald, M., Dobson, V., Sebris, S. L., Baitch, L., Varner, D., \& Teller, D. Y. (1985). The acuity card procedure: A rapid test of infant acuity. Investigative Ophthalmology and Visual Science, 26, 1158-1162.

Norcia, A. M., Clarke, M., \& Tyler, C. W. (1985). Digital filtering and robust regression techniques for estimating sensory thresholds from the evoked potential. Institute of Electrical and Electronics Engineers Engineering in Medicine and Biology, 4, 26-38.

Norcia, A. M., \& Tyler, C. W. (1985). Spatial frequency sweep-VEP: Visual acuity during the first year of life. Vision Research, 25, 1399-1408.

Norcia, A. M. (1994). Vision testing by visual evoked potential techniques. In S. J. Isenberg (Ed.), The eye in infancy (pp. 157-173). St. Louis, MO: Mosby-Year Book.

Regan, D. (1973). Rapid objective refraction using evoked brain potentials. Investigative Ophthalmology, 12, 669-703.

Riddell, P. M., Landenheim, B., Mast, J., Catalano, T., Nobile, R., \& Hainline, L. (1977). Comparison of measures of visual acuity in infants: Teller acuity cards and sweep visual evoked potentials. Optometry and Visual Science, 74, 702-707.

Salomão, S. R., \& Ventura, D. F. (1995). Large scale population age norms for visual acuities obtained with Vistech/Teller acuity cards. Investigative Ophthalmology and Visual Science, 36, 657-670.

Schroeder, C. E. (1991). Striate cortical contribution to the surface-recorded pattern-reversal VEP in the alert monkey. Vision Research, 31, 1143-1157. 


\section{Desenvolvimento da Acuidade Visual de Grades}

Shimojo, S., Paradiso, M., \& Fujita, I. (2001). What visual perception tells us about mind and brain. Proceedings from the National Academy of Science, 98, 12340-12341.

Skarf, B. (1989). Clinical use of visual evoked potentials. In D. Fuller \& D. Birch (Eds.), Ophthalmology clinics of North America (pp. 499-518). Philadelphia: W.B. Saunders Co.

Skoczenski, A. M., \& Norcia, A. M. (2002). Late maturation of visual hyperacuity. Psychological Science, 13, 537-541.

Sokol, S. (1978). Measurement of infant visual acuity from pattern reversal evoked potentials. Vision Research, 18, 33-39.

Sokol, S., Hansen, V. C., Moskowitz, A., Grenfield, P., \& Towle, V. L. (1983). Evoked potential and preferential looking estimates of visual acuity in pediatric patients. Ophthalmology, 90, 552-562.

Sokol, S., Moskowitz, A., Mccormack, G., \& Augliere, R. (1988). Infant grating acuity s temporally tuned. Vision Research, 28, 1357-1366.

Teller, D. Y, Morse, R., Borton, R., \& Regal, D. (1974). Visual acuity for vertical and diagonal gratings in human infants. Vision Research, 14,1433-1439.

Teller, D. Y. (1979). The forced-choice preferential looking procedure: A psychophysical technique for use with human infants. Infant Behavioral Development, 2, 135-143.

Teller, D. Y. (1997). First glances: The vision of infants. The friedenwald lecture. Investigative Ophthalmology and Visual Science, 38, 2183-2203.

Tyler, C. W., Apkarian, P. A., \& Levi, D. M. (1979). Rapid assessment of visual function: An electronic sweep technique for the pattern VEP. Investigative Ophthalmology and Visual Science, 18, 703-712.

Recebido em: 14.07.2004

Aceito em: 7.10.2004 
